\title{
Developing the field of neurogenetics
}

\author{
Ulrich Müller $^{1}$ • Georg Auburger ${ }^{2}$ - Manuel B. Graeber ${ }^{3}$ - Louis J. Ptacek ${ }^{4}$
}

Published online: 21 November 2017

(C) Springer-Verlag GmbH Germany, part of Springer Nature 2017

"Neurogenetics" studies the genetic basis of normal and abnormal differentiation and function of the nervous system. The field was pioneered by Seymour Benzer who investigated behavior and neurodegeneration in Drosophila. His work has also increased our knowledge of genetic aspects of neuronal (dys-) function in other organisms including humans. Such knowledge is a prerequisite for a better understanding of neurogenetic diseases which are defined as "clinical disease(s) caused by a defect in one or more genes which affect the differentiation and function of the neuroectoderm and its derivatives" [1]. Since the mid1980 s of the last century, the advent of positional cloning techniques has facilitated direct approaches to the elucidation of neurogenetic disorders, temporarily overshadowing the relevance of the study of model organisms.

The rapid identification of gene defects in monogenic diseases such as Huntington disease, ataxias, dystonias, and many others prompted the inception of the journal Neurogenetics in 1997 as a forum for the communication and discussion of findings in the field. During its first 20 years of existence, the journal has mainly focused on monogenic neurological disorders in humans and occasionally reported relevant findings in other organisms. Specifically, Neurogenetics has published experiments towards

Ulrich Müller

Ulrich.Mueller@humangenetik.med.uni-giessen.de

1 Institut für Humangenetik, Giessen University, Schlangenzahl 14, 35392 Gießen, Germany

2 Neurology, Goethe University Medical School, 60590 Frankfurt am Main, Germany

3 Brain and Mind Centre, University of Sydney, 94 Mallet St, Camperdown, NSW 2050, Australia

4 Department of Neurology, University of California, San Francisco, USA a better understanding of pathological mechanisms caused by mutations in single genes. During recent years, observations in "complex" neurological disorders, i.e., diseases caused by alterations in multiple genes and by environmental factors, have become more prominently presented in the Journal.

At this junction, Ulrich Müller, a founding editor of the Journal, is stepping down at the helm of Neurogenetics and Georg Auburger follows him as an editor. In the coming years, we expect an increase in publications on findings in the common "complex" neurological disorders such as degenerative neural processes and epilepsies.

The study of the function of genes contributing to the common neuronal diseases will be expedited by the use of cell lines (including induced pluripotent stem and neuronal cells) and model organisms such as Drosophila, and by the advent of highthroughput sequencing techniques. Epigenetic phenomena such as abnormal DNA methylation and histone acetylation are becoming increasingly recognized as important pathogenic factors in neurological disorders as are the importance of RNA regulation and toxicity. Furthermore, it is now becoming possible to untangle the complexities of gene and brain function that result in normal and abnormal behavior and contribute to intelligence and emotions. The Journal is determined to cover these novel trends and explicitly encourages submission of papers on the function of genes implicated in common neurological diseases and traits. We anticipate that these functional studies will point towards causative therapies which shall be reported in Neurogenetics.

Given the many ongoing thrilling developments in the field, we are looking forward to many more exciting years of reporting groundbreaking discoveries in Neurogenetics.

Ulrich Müller, Giessen, Germany Georg Auburger, Frankfurt, Germany Manuel B. Graeber, Sydney, Australia Louis J. Ptacek, San Francisco, CA, USA November 2017 
Thank you, Professor Ulrich Müller, and Welcome, Professor Georg Auburger!

The journal Neurogenetics was founded in 1997 by Professor Ulrich Müller and Professor Manuel Graeber and Springer has been fortunate to publish the journal since 1998. During the first years of publication, the Journal quickly became a preferred venue for publishing findings that contribute to a better understanding of the genetic basis of normal and abnormal functions of the nervous system.

On behalf of Springer, I would like to warmly thank the outgoing Founding Editor, Ulrich Müller, for his longstanding commitment to Neurogenetics as well as for his energy and vision to found a new journal as soon as the new research field had emerged. Over the past decades, Ulrich Müller has been instrumental in advancing the field in Germany as well as internationally.

And I would like to warmly welcome Professor Georg Auburger to the team of Editors-in-Chief for Neurogenetics. Over the past years, Georg Auburger has strongly supported the journal as a referee and author. We at Springer are truly honored to work with him and the whole editorial team. The field of neurogenetics is constantly moving, especially with the novel technologies and possibilities to study complex, multi-gene diseases. There is little doubt that neurogenetics will continue to flourish as a field for decades to come and we look forward to a bright future for Neurogenetics. With this in mind, the editors and publishers of Neurogenetics offer their best wishes to past, present and future authors, reviewers and readers of the journal.

\section{Andrea Pillmann, Executive Editor, Springer Heidelberg, Germany \\ November 2017}

\section{Reference}

1. Müller U, Graeber MB (1996) Neurogenetic diseases: molecular diagnosis and therapeutic approaches. J Mol Med (Berlin) 74(2): 71-84. https://doi.org/10.1007/BF00196782 